\title{
The Utility of Deformable Image Registration for Small Artery Visualisation in Contrast-Enhanced Whole Body MR Angiography
}

\author{
Daniel Foley \\ Trinity College Dublin, Ireland \\ Jacinta Browne \\ Technological University Dublin, jacinta.browne@tudublin.ie \\ Xiahai Zhuang \\ Centre for Medical Image Computing, Department of Medical Physics and Bioengineering, University \\ College London
}

See next page for additional authors

Follow this and additional works at: https://arrow.tudublin.ie/scschphyart

Part of the Biological and Chemical Physics Commons

\section{Recommended Citation}

Foley, D. et al. (2014) The utility of deformable image registration for small artery visualisation in contrastenhanced whole body MR angiography. Physica Medica: European Journal of Medical Physics (2014), Dec;30(8):898-908. doi:10.1016/j.ejmp.2014.08.001

This Article is brought to you for free and open access by the School of Physics \& Clinical \& Optometric Science at ARROW@TU Dublin. It has been accepted for inclusion in Articles by an authorized administrator of ARROW@TU Dublin. For more information, please contact arrow.admin@tudublin.ie, aisling.coyne@tudublin.ie, gerard.connolly@tudublin.ie.

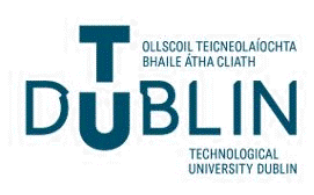




\section{Authors}

Daniel Foley, Jacinta Browne, Xiahai Zhuang, Barry Sheane, Dearbhail O'Driscoll, Daniel Cannon, Niall Sheehy, James Meaney, and Andrew Fagan 


\section{The utility of deformable image registration for small artery visualisation in contrast-enhanced whole body MR angiography}

Daniel Foley ${ }^{1}$, Jacinta E Browne ${ }^{2}$, Xiahai Zhuang ${ }^{3,4}$, Barry Sheane ${ }^{1}$, Dearbhail O’Driscoll ${ }^{1}$, Daniel Cannon ${ }^{1}$, Niall Sheehy ${ }^{1}$, James F Meaney ${ }^{1}$, Andrew J Fagan ${ }^{1 *}$

${ }^{1}$ National Centre for Advanced Medical Imaging (CAMI), St. James's Hospital, Dublin / School of Medicine, Trinity College Dublin, Ireland

2 Medical Ultrasound Physics Group, School of Physics / IEO \& FOCAS Institutes, Dublin Institute of Technology, Kevin's Street, Dublin 8, Ireland,

${ }^{3}$ Centre for Medical Image Computing, Department of Medical Physics and Bioengineering, University College London

${ }^{4}$ Shanghai Advanced Research Institute, Chinese Academy of Sciences, Shanghai, China

\section{* Corresponding Author:}

Andrew J Fagan,

National Centre for Advanced Medical Imaging (CAMI),

Room MRI 11,

St. James's Hospital,

Dublin 8,

Ireland

Tel: $+353-18964331$

Email: faganan@tcd.ie 


\section{Abstract}

\section{Purpose}

An investigation was carried out into the effect of three image registration techniques on the diagnostic image quality of contrast-enhanced magnetic resonance angiography (CE-MRA) images.

\section{Methods}

Whole-body CE-MRA data from the lower legs of 27 patients recruited onto a study of asymptomatic atherosclerosis were processed using three deformable image registration algorithms. The resultant diagnostic image quality was evaluated qualitatively in a clinical evaluation by four expert observers, and quantitatively by measuring contrast-to-noise ratios and volumes of blood vessels, and assessing the techniques' ability to correct for varying degrees of motion.

\section{Results}

The first registration algorithm ('AIR') introduced significant stenosis-mimicking artefacts into the blood vessels' appearance, observed both qualitatively (clinical evaluation) and quantitatively (vessel volume measurements). The other two algorithms ('Slicer' and 'SEMI') based on the normalised mutual information (NMI) concept and designed specifically to deal with variations in signal intensity as found in contrast-enhanced image data, did not suffer from this serious issue but were rather found to significantly improve the diagnostic image quality both qualitatively and quantitatively, and demonstrated a significantly improved ability to deal with the common problem of patient motion.

\section{Conclusions}

This work highlights both the significant benefits to be gained through the use of suitable registration algorithms and the deleterious effects of an inappropriate choice of algorithm for contrast-enhanced MRI data. The maximum benefit was found in the lower legs, where the small arterial vessel diameters and propensity for leg movement during image acquisitions posed considerable problems in making accurate diagnoses from the un-registered images.

\section{Keywords}

Deformable image registration, Atherosclerosis . Peripheral Arterial Disease - Whole Body MR Angiography, Contrast Enhanced, Normalised Mutual Information 



\section{Introduction}

Peripheral arterial disease (PAD) is a manifestation of cardiovascular disease in the extremities of the body with reported incidences of $11-16 \%$ in persons over 55 years old [1]. PAD has been linked to a 6-fold increase in risk for myocardial infarction [2] and an at least 2-fold rise in the risk of ischaemic stroke [3], and hence the detection of this condition is critical for at-risk patients [4]. PAD involves the build-up of plaque in the arteries (a condition called atherosclerosis), and although patients usually present with a stenosis in one area (and hence diagnostic imaging tends to focus exclusively on that symptomatic area), it is likely that the disease will be manifest asymptomatically elsewhere in the body. The entire arterial system can, however, be investigated using a whole body contrast-enhanced magnetic resonance angiography (CE-MRA) approach, with a view to determining the extent of the disease and whether any asymptomatic PAD can be revealed. Whole body CE-MRA uses a moving table technique wherein the body is sub-divided into 4 or 5 "stations" which are sequentially scanned by automatically moving the anatomy covered by each station into the isocentre of the magnet. Initially, pre-contrast images are acquired at each station, followed by the rapid injection of a bolus of contrast agent and a re-acquisition of all images while chasing the bolus down the body. The respective pre- and post-contrast images are subtracted to accentuate visualisation of the arterial system.

However, mis-registration artefacts can cause significant deterioration of the resultant image quality, particularly evident when trying to visualise small vessels. This can occur due to patient motion, but also due to mis-alignment of the table following its movement in and out of the magnet between image acquisitions. In the bolus-chase method, the pre-contrast images are acquired feet first and finishing at the head, so that the post-contrast imaging commences where the contrast agent arrives first (i.e. upper body regions) and is chased down the body thereafter. As such, several minutes pass between the acquisition of the pre- and post-contrast images, particularly of the lower legs, while

furthermore the table has twice moved the full extent of the body between acquisitions. Leg immobilisation techniques go some way towards preventing patient movement artefacts but do not eliminate this completely, particularly for patients with advanced PAD who have difficulty remaining still.

Image registration techniques are well established for dealing with patient movement artefacts, although routine clinical MRI applications focus predominately in the brain where robust registration techniques exist for a wide range of applications such as diffusion weighted/tensor imaging [5], functional MR [6], and serial imaging [7, 8]. Nevertheless, this remains an area of active research, with the continued evolution of registration algorithms tailored for specific applications even within 
the brain [9] or between imaging modalities (for example, MRI and PET [10]). For an overview of the literature in MRI and other imaging modalities, including areas outside of the brain, the reader is referred to a recent comprehensive review [11].

Conventionally, registration techniques work through a direct analysis of pixel signal intensities or by identifying features within the two images to be registered, and hence a common assumption is that both images have the same signal intensities in corresponding anatomical structures which are simply displaced somewhat relative to each other. The earliest generation of registration algorithms were based on this rather simplistic approach, an example of which is the Automated Image Registration (AIR) algorithm originally described by Woods et al in 1992 [12] and still widely used today [9, 13, 14].

An added complexity arises, however, in studies involving the administration of contrast agents whose purpose is specifically to change the localised signal intensity, whether in tumours in the case of dynamic contrast enhanced (DCE) studies [15], or blood vessels in CE-MRA examinations, rendering inaccurate the assumption of no signal changes between common image features the images to be registered. To this end, a new generation of registration techniques have been developed for application in DCE studies specifically to deal with such image intensity changes. The first such example was reported by Reuckert et al. in 1999 in a DCE study in the breast, where both signal intensity changes due to contrast agent and non-rigid tissue deformations were successfully corrected using a novel registration algorithm based on the concept of normalised mutual information (NMI) [16]. NMI stems from the field of information theory and expresses the amount of information that one image contains about another. Simply put, NMI postulates that two images will be optimally aligned when one of the images is "definable" by the other, in other words when the (normalised) mutual information between them is maximised. Hence, the registration problem reduces to shifting pixels such that the NMI between the resulting images is maximised. This approach is thus capable of dealing with signal intensity differences, and furthermore has been shown to accurately and robustly align images from different modalities where variable signal intensities may be expected [17]. It has been used in several DCE studies to good effect, including studies in the breast [18-20], lung [21], kidney [22], liver [23] and prostate [24]. A recently reported improvement on the mutual information approach involved introducing spatial information into the registration scheme, wherein the original image is sub-divided into an array of local regions, with the MI-maximisation procedure performed separately and independently over each sub-region [25]. This method demonstrated significant improvements in performance in brain, cardiac and contrast-enhanced liver datasets. 
Many registration techniques designed to deal with signal intensity changes due to contrast agent uptake suffer from a particular misregistration artefact wherein enhancing regions undergo a volume change after the registration step [26]. Efforts to minimise this effect have focussed on imposing constraints on the allowable deformation, although this was achieved at the expense of the motioncorrection performance [19]. A recent study comparing three registration algorithms found that an algorithm based on sequential elastic registration best preserved tumour volume and shape in 16 tumours distributed among the lung, liver, uterus and chest wall [27].

Despite the potential to improve the diagnostic image quality of CE-MRA images using registration techniques, there are very few studies reported in the literature in this field. Hipwell et al. reported a technique for registering MRA images with those obtained with X-ray digital subtraction angiography, however the aim was to provide complementary information from both modalities and no improvement was reported in the native MRA image quality [28]. In a separate study, an improvement in image quality was reported in CE-MRA images in the lower legs following registration using the AIR algorithm, with non-linear (deformable) registration found to perform slightly better than more simple linear approaches [29]. It should be noted that this algorithm was not intended to be used to register images with differing signal intensity values (as occurs, for example, when one of the images was acquired with a contrast agent in the vessels). To date, no applications of image registration algorithms designed specifically to deal with signal intensity changes in contrastenhanced studies have been reported in CE-MRA.

The aim of the current study was to perform a critical evaluation of the performance of two NMIbased deformable registration algorithms for CE-MRA data in comparison to that of the AIR algorithm used in the previous study [29]. It was hypothesised that the AIR algorithm may not be suitable for this specific application, and that superior performance would be achieved with NMIbased algorithms, in particular with a novel NMI algorithm designed to incorporate spatial information into the registration scheme. Changes in image quality following application of each registration algorithm were evaluated qualitatively and quantitatively via a clinical evaluation of the diagnostic image quality and measurements of the contrast-to-noise ratio respectively. To check for inadvertent volume changes in enhancing regions, blood vessel volumes were also compared over identical sections in images generated using each algorithm. 


\section{Materials and Methods}

\section{Data Acquisition}

A protocol was devised to acquire very high spatial and temporal resolution CE-MRA data of the first pass of a single bolus of contrast agent through the entire arterial system in patients with asymptomatic atherosclerosis. The use of a single bolus of contrast agent was mandated by the desire to minimise the risk of nephrotic systemic fibrosis in the patient cohort. Images from 27 patients (average age: 57.8 years, 19 female) recruited onto the study were acquired using a 3 T MRI system (Achieva, Philips Medical Systems, The Netherlands) located in the National Centre for Advanced Medical Imaging (CAMI) in St. James's Hospital / Trinity College Dublin. Approval for the study was obtained from the local Institutional Review Board (Ethics Committee, St. James's Hospital, Dublin) and informed consent was obtained from all patients.

The acquisition protocol centres on a $\mathrm{T}_{1}$-weighted $3 \mathrm{D}$ fast gradient echo sequence with a 5 -station protocol comprising: head/neck, chest/abdomen, pelvis, upper legs, and lower legs. The images were acquired in a coronal orientation using the MR system's body coil for signal reception, resulting in an isotropic spatial resolution of either 0.9 or $1.5 \mathrm{~mm}^{3}$ depending on the station (full details of the acquisition parameters are presented in Table 1). A partial Fourier data acquisition scheme was used to maximise the acquisition speed (halfscan factor $=0.625$ ). The centre of $\mathrm{k}$-space was acquired at the beginning of each scan using the CENTRA (Contrast ENhanced Timing Robust Angiography) [30] technique, timed so that the acquisition of the k-space centre data was correlated with the peak of the contrast agent bolus passing through that region. Thirteen of the patients were given a single-bolus injection of $10 \mathrm{ml}$ of Vasovist (Bayer Schering Pharma AG, Germany), with $2.5 \mathrm{ml}$ injected at a rate of $0.5 \mathrm{ml} / \mathrm{s}$ followed by the remaining $7.5 \mathrm{ml}$ at $0.25 \mathrm{ml} / \mathrm{s}$. The injection of a $40 \mathrm{ml}$ saline flush was then administered at a rate of $0.25 \mathrm{ml} / \mathrm{s}$. During the course of the study, this agent was no longer commercially available, and hence the remaining fourteen patients were given a single-bolus injection of $20 \mathrm{ml}$ of Multihance (Bracco, Milan, Italy), with $8 \mathrm{ml}$ injected at $0.8 \mathrm{ml} / \mathrm{s}$ followed by $12 \mathrm{ml}$ injected at $0.5 \mathrm{ml} / \mathrm{s}$ and a $40 \mathrm{ml}$ saline flush injected at $1.0 \mathrm{ml} / \mathrm{s}$. For both contrast agents, the respective injection protocols were developed through prior experience with these agents to optimise the resultant contrast in the vessels. No differences in enhancement profiles for each contrast agent were noted by any of the 4 expert observers. The patients' legs were strapped together and secured with foam material to restrict leg movement as much as possible.

Pre-contrast images were acquired starting from the lower leg station moving upwards to the head station. These images were used as mask images which were subtracted from the post-contrast images. Once the contrast was injected, the post-contrast image acquisition began at the head station 
and proceeded down to terminate at the lower leg station. At the beginning of the acquisition of the post-contrast images, a Smart Tourniquet (Topspins Inc., MI, USA) fitted around both thighs was inflated to reduce the blood flow to the lower legs, thereby minimising the appearance of venous enhancement in the images [31]. Total post-contrast image acquisition time was 106 seconds, including the four separate $4 \mathrm{~s}$ table movements that were required between stations.

\section{Image Registration}

The current study focussed on investigating the performance of deformable registration algorithms on improving the visibility of the small diameter vessels in the lower legs, since prior experience of image registration with MRA data indicated that no significant benefit was to be gained by performing deformable registration outside the lower legs (although performing a rigid registration was found to improve the diagnostic image quality in the upper legs and pelvis). Three deformable algorithms were investigated (henceforth referred to as AIR, Slicer and SEMI), as described in the following:

Automatic image registration (AIR): One of the first widely-available deformable algorithms, this method was used in the only study reported to date on the use of deformable registration in CE-MRA [29], and hence was used as the reference method against which the newer methods were compared (AIR version 5.2.5, http://bishopw.loni.ucla.edu/AIR5/). The alignment technique used by AIR is based on the idea that when the two images are correctly aligned, the difference between corresponding voxel values is equal to a single multiplicative factor. If the images are not correctly aligned, this factor will vary throughout the image on a voxel by voxel basis. Therefore, the algorithm attempts to minimise this variation in order to achieve the maximum alignment possible. Details of its functionality are available in the original 1992 publication by Woods et al. describing this algorithm [12].

3D Slicer: This open source software environment (version 3.6, http://www.slicer.org/) implements the Insight Segmentation and Registration Toolkit (ITK) (www.itk.org) and uses a deformable registration algorithm originally developed by Rueckert et al. [16] to register contrast-enhanced breast MR images using a free-form deformation (FFD) model based on B-splines to describe the local breast motion. The choice of Slicer was based on its wide availability and its use of NMI as the similarity metric, hypothesised to better deal with the contrast agent-mediated signal intensity changes within the MRA image data. This worked by defining a mesh (labelled $\Phi$ ) of control points $\phi_{i, j, k}$ with a uniform spacing that are placed throughout the image volume $\Omega$. The resolution of the mesh dictates the degree of non-rigid deformation that can be modelled using $\Phi$ as the transformation 
parameter. As a result, increasing the number of control points will increase the number of degrees of freedom but also increase the computational complexity of the algorithm. To achieve the best compromise between these, a hierarchical multi-resolution approach was used. This involved beginning with a relatively low control point mesh resolution which was used to compute the local transformation. Once completed, the distance between the control points of $\Phi^{l}$ and $\Phi^{l+1}$, where $\Phi^{l+l}$ is the subsequent iteration of $\Phi^{l}$, was decreased which results in a higher mesh resolution for $\Phi^{l+1}$. The normalised mutual information metric, which is well suited to contrast-enhanced data, was then used as the similarity metric to measure the alignment between the two images. To calculate the required transformation, the Slicer algorithm determined the optimal affine transformation parameters by maximising $C_{\text {similarity, }}$, defined as:

$$
C_{\text {similarity }}(A, B)=[H(A)+H(B)] / H(A, B)
$$

where $A$ and $B$ denote the images, and $H$ is the relevant entropy measure of the dispersion of information in the images. This accounted for any relative global motion between the two images. It then iteratively computed the optimal transformation of the control point mesh that was used for the non-rigid deformations by adjusting the cost function that provided an optimal compromise between maximising the NMI and keeping the transformation smooth.

Spatially Encoded Mutual Information (SEMI): This deformable registration algorithm was recently developed by Zhuang et al. [25] with the specific aim of dealing with images which have different intensity distributions in local regions, such as dynamic contrast enhanced data, and hence the interest was to compare its performance against the baseline AIR and NMI-based Slicer algorithms. The SEMI algorithm, which also uses NMI as the similarity metric, attempts to overcome some limitations of standard NMI-based approaches by dividing the images into small sub-regions and computing the associated entropy measures of each sub-region separately, while also introducing spatially-varying weighting factors for each voxel within the sub-region in order to modulate the resulting deformation for each voxel depending on its position. The weighting factors that were used monotonically decreased with distance from the voxel position. This allowed the algorithm to increase the accuracy of the registration process by using the spatial information present in the images. The transformation parameters were then iteratively altered using a local ascent optimisation. This optimisation scheme uses only the FFD control point associated with the spatial location of the entropy measure and excludes all others, which significantly decreases the computational time needed by using only these local control points but adjusting every registration parameter during each iteration. Further details of this algorithm are available in [25]. 
The time required by each algorithm to perform the registration was recorded based on the use of a standard desktop computer with a $2.4 \mathrm{GHz}$ quad core CPU and $4 \mathrm{~GB}$ of RAM.

\section{Clinical Evaluation}

The unregistered and registered images were clinically evaluated in an independent and blinded manner by four expert observers (three radiologists ranging from 2 to 28 years' experience, and one imaging physicist (AJF) with 17 years' experience), with the images loaded onto a computer workstation with a high-quality monitor (Extended MR WorkSpace, Philips Medical Systems, The Netherlands). A display protocol was set-up which loaded the four datasets in a randomised manner into one of 4 viewing windows, wherein each window could display either the native subtracted images or the maximum intensity projection (MIP) of the respective datasets (that is, MIPs of the unregistered or three registered datasets). The settings within each viewing window were linked, allowing the observers to adjust the brightness and contrast levels, the image magnification, and the slice position displayed, in a co-ordinated fashion across all 4 windows simultaneously.

The observers were asked to rank the images based on overall diagnostic image quality, paying particular attention to the effect on vessel integrity/sharpness, the loss of any small vessels following registration, the ability to delineate stenoses, the degree of background tissue suppression, and the number and severity of artefacts evident in the images. The four images from each patient were independently ranked by each observer on a scale of 1 - 4 (where 1 was best).

\section{Contrast-to-Noise Ratio Measurement}

Contrast-to-noise ratio (CNR) measurements were compared between the registered and unregistered images in order to quantify the performance of the algorithms in removing background tissue while maintaining signal intensity in the vessels themselves. To this end, regions of interest (ROIs) were manually defined in the poplitaeal artery and in the immediately surrounding tissue for all patients; the ROIs were drawn on the MIP images from the unregistered datasets and copied to the corresponding registered datasets. The poplitaeal artery was selected for this purpose since it was possible to consistently draw a ROI of reasonable size within this artery while avoiding the arterial wall. A typical ROI placement is shown in Figure 1, although in four patients the field of view scanned necessitated drawing the ROI in the anterior tibial artery. Corresponding background tissue ROIs were drawn within a distance of 5 voxels from the artery. 
The contrast-to-noise ratio was calculated using $C N R=\left(S I_{\text {Artery }}-S I_{\text {Tissue }}\right) / S D_{\text {Tissue }}$, where $S I_{\text {Artery }}$ and $S I_{\text {Tissue }}$ refer to the average signal intensity in the artery and background tissue ROIs, respectively, while $S D_{\text {Tissue }}$ refers to the standard deviation in the background tissue ROI.

\section{Blood Vessel Volume Measurements}

The integrity of vessel reproduction is essential in MRA, and hence any artificial reduction in vessel volume following the registration process could have significant clinical implications. To investigate the degree to which this occurred, if at all, for any of the registration algorithms used in this study, the volume of carefully-defined sections of the anterior tibial artery were measured in the registered and unregistered datasets. To do this, a section of the artery was delineated by defining an upper and lower cut-off plane, orientated perpendicular to the artery, in the unregistered image dataset, which were subsequently copied to the corresponding registered datasets. The artery was then segmented within these planes by selecting a seed point within the artery section and using a segmentation algorithm to propagate a volume of interest (VOI) through the vessel until the upper and lower bounds were reached. This was done using the ITK-SNAP software, version 2.2 (http://www.itksnap.org), which uses an active contour segmentation algorithm implemented using level set methods to grow the initial seed volume (placed in the centre of the artery's lumen) circularly out to the edges of the artery and longitudinally until the boundary planes are reached [32]. All segmented volumes were visually inspected to ensure no bleeding of the segmented volume into the background tissue occurred; however, in all cases, the high contrast of the contrast-enhanced blood vessels relative to the surrounding background tissue, even in the unregistered datasets, lead to a successful segmentation of the vessel.

\section{Movement Correction Performance}

Another important feature of registration algorithms is the degree of movement that they can successfully correct for. This is of particular concern when imaging the legs of patients suffering from PAD, who often have extreme difficulty lying still and consequently one often observes significant movement of one leg relative to the other. To investigate this, a phantom mimicking the major arteries of the lower legs was constructed. The phantom was made using a 3\% agar gel, doped with $0.1 \mathrm{mM}$ of $\mathrm{MnCl}_{2}$ to mimic the relaxation properties of tissue, and with embedded vessels of internal diameter $3.2 \mathrm{~mm}, 2.4 \mathrm{~mm}$, and $1.6 \mathrm{~mm}$ through which the same concentration of contrast agent as used in the clinical study was introduced. The phantom was scanned using the same protocol as outlined earlier, with varying shifts between the pre- and post-contrast images (ranging from 0 to $25 \mathrm{~mm}$ in $1 \mathrm{~mm}$ steps in the direction perpendicular to the vessels).

\section{Statistical Analyses}


Statistical analysis was performed in SPSS (version 21, IBM Corporation, USA) using the Friedman nonparametric two way analysis of variance (ANOVA) on ranks with the algorithm used as the factor for the clinical evaluation, while a paired-samples t-test was used to analyse the CNR and vessel volume for each algorithm. A value of $p<0.05$ was set as the criterion for statistical significance.

\section{RESULTS}

The average time taken for each algorithm to register the images was 36.7, 0.9, and 33.1 minutes for AIR, Slicer, and SEMI respectively. It should be noted that the SEMI technique was not yet optimised for speed, and in fact only used one core of the central processing unit (CPU) on the computer, so it is expected that significant improvements on the processing speed will be achieved in future versions of this software. Nevertheless, the average time of only 54 seconds for Slicer to process the images demonstrates the practicability of performing this relatively complex computational process, aided by efficient algorithm design and modern computing power (even on a very standard desktop computer).

Before the images could be submitted for the clinical evaluation, it was necessary to remove an edge artefact introduced by all registration algorithms. These artefacts were most prominent at the anterior and/or posterior edges of the subtracted image volume, and originated from the registration process encountering edges of the image volume - in these locations, the (3D) algorithms were most likely forced to extrapolate the deformation field to regions outside the images field of view where no pixel information exists, resulting in a warped deformation field near the edge and hence to the observed artefacts. They were easily removed by deleting the edge slice(s) from the subtracted image stack before generating the MIP images. An example of this artefact is presented in Figure 2, which shows the manifestation of the artefact in the first slice of the subtracted image stack, together with the corresponding uncorrected and corrected MIPs . Although all three algorithms produced this artefact in all patients (with Slicer producing no artefact in two patient datasets), its manifestation was least prevalent for the Slicer algorithm which required the least intervention. Care was taken to ensure that all CNR measurements were performed on the images after the removal of any slices containing these prominent subtraction artefacts, while background ROIs delineating the 'noise' component were placed to avoid any further subtraction artefacts in the images.

\section{Clinical Evaluation}


The results from the blinded clinical evaluation of the 27 patient datasets are presented in Table 2 . In all cases, it was felt that the three registration algorithms significantly improved the diagnostic image quality compared to the unregistered images; an example of this improvement is presented in Figure 3. SEMI was found to produce a significant improvement compared to AIR ( $p<0.01)$, and although Slicer improved the quality compared to AIR in $74.1 \%$ of reads (80/108) the improvement was not significant $(\mathrm{p}=0.09)$. When compared to Slicer, the SEMI technique improved the quality in only $33.3 \%$ of reads, with an equal ranking given in $54.6 \%$ of reads, hence the improvement was not significant $(\mathrm{p}=0.19)$. Table 3 presents the kappa values illustrating the degree of interobserver agreement. The relatively small differences between the Slicer- and SEMI-produced images in many cases was reported to make the categorisation difficult and may account for the less than perfect agreement.

It is interesting to note that AIR was found to perform best among the registration algorithms with regards to general background tissue signal suppression; an example is shown in Figure 4, where the different processing methods are compared for the left leg of one patient. However, all observers also commented on a quite noticeable deleterious effect that the AIR algorithm had on the appearance of the blood vessels in all patient images examined, wherein the vessel integrity appeared to be severely compromised in many instances, and in fact this was cited as the primary reason why AIR was ranked worse than the other two registration algorithms. Examples of these artefacts introduced by AIR are presented in Figure 5, which compares the unregistered and registered MIP images for one representative patient.

\section{Quantitative Measurements}

Results from the quantitative measurements are presented in Table 4, in which differences in the mean (and standard deviation) contrast-to-noise ratio and vessel volumes between the unregistered and three registration algorithms are shown together with p-values indicating the significance of the differences across the 27 patient datasets. The CNR difference measurements reflect the findings of the clinical evaluation in that all three registration algorithms improved the CNR significantly $(\mathrm{p}<0.05)$. Interestingly, despite the superior background tissue suppression reported by the expert observers for AIR, its CNR values were lower than those of Slicer in $74 \%$ of cases (20/27), although the difference was not significant at a 95\% confidence level $(p=0.066)$. However, the SEMI algorithm performed significantly better than both AIR $(\mathrm{p}<0.01)$ and Slicer $(\mathrm{p}<0.014)$

The clinical evaluation findings were also reflected in the measurements of the vessel volumes: while those of Slicer and SEMI were unchanged compared to the un-registered images, AIR was found to introduce a significant decrease in vessel volume $(\mathrm{p}<0.001)$, with an average decrease of $17.9 \%$ 
compared to the unregistered images. An example of this artefact is presented in Figure 6, in which the segmented vessel volumes at the location of the branching of the poplitaeal and anterior tibial arteries are compared for the raw and registered datasets; the artefactual narrowing of the anterior tibial artery caused by the AIR algorithm, as seen in Figure 6(b), could potentially lead to a misdiagnosis in this patient. No decrease in volume was produced by either Slicer or SEMI, demonstrating the fidelity of registration produced for these contrast-enhanced structures.

\section{Movement Correction Performance}

AIR was the first algorithm to struggle with the motion challenge, which manifested as the disappearance of the $1.6 \mathrm{~mm}$ diameter vessel when the phantom was displaced by a mere $1 \mathrm{~mm}$ between the pre- and post-contrast image acquisitions (Figure 7(a)). The background suppression remained constant for all motions investigated.

In images produced by the Slicer algorithm, artefactual white dots appeared in the registered images following $6 \mathrm{~mm}$ of movement, increasing in prevalence with increasing movement, while the background suppression began to fail above $10 \mathrm{~mm}$ of movement, particularly towards the centre of the images (Figure 7(b)). At $11 \mathrm{~mm}$ and above, a further artefact was noted, manifested as a mirror vessel appearing next to a real vessel, as illustrated for the $3.2 \mathrm{~mm}$ diameter vessel in Figure 7(b), indicating that the algorithm did not successfully associate the pre- with the post-contrast vessel. Similar artefacts appeared next to the smaller diameter vessels for larger lateral movements. These various artefacts, while fairly obvious in this simplified phantom model, could pose problems in a clinical image dataset where the more complex vasculature / anatomy would complicate their identification.

The SEMI algorithm performed best, and was found to work well even with the maximum attempted displacement of $25 \mathrm{~mm}$ with all vessels faithfully retained, although some spotting artefacts and a slight deterioration of the background suppression occurred for displacements of $15 \mathrm{~mm}$ and above (Figure 7(c)). Table 5 summarises the image artefact manifestation as a function of displacement for the three registration algorithms, illustrating the robustness of the SEMI algorithm against motion.

\section{Discussion}


The key finding of this paper is that the image quality of CE-MRA images may be significantly improved using readily-available off-the-shelf registration techniques, although care must be exercised in the choice of registration algorithm used. This is borne out by the finding herein that registration artefacts were introduced by a conventional algorithm used in a previous MRA study but, critically, which was not designed to deal with the signal intensity increase in blood vessels caused by the introduction of the contrast agent. A new generation of algorithms have been developed over the past decade to address this specific contrast-enhancing problem yet they have not, to date, been explored for use with MRA image data. This study demonstrates for the first time the significant improvement in diagnostic image quality to be gained from their use.

The AIR algorithm was originally developed to register brain images between positron emission tomography (PET) and MRI, and the method it employs to perform the registration (based on anticipated signal intensity ratio variations for brain tissue from both modalities, the variance of which it attempts to minimise) is clearly not suitable for CE-MRA data. Despite producing reasonably good background tissue suppression across the image as a whole, the algorithm's inability to deal with the contrast-enhanced vessels was manifest in both the introduction of artefacts in the vessels' appearance, which could quite easily hide a real stenosis or lead to a misclassification of a stenosis, and its poor contrast-to-noise performance close to the vessel themselves. Furthermore, its inability to deal with vessel displacements in excess of $1 \mathrm{~mm}$ is of concern, given that motions of this magnitude can be expected in a clinical whole-body MRA study [29]; the disappearance of a small artery could easily be misdiagnosed as a blockage.

The results from the current study demonstrate that the use of algorithms based on the normalised mutual information metric such as Slicer and SEMI are suitable for registering CE-MRA images. Both algorithms worked consistently well for all patient data investigated, producing significant improvements in diagnostic image quality throughout the images. The SEMI algorithm is a recent improvement on the NMI approach, and its inclusion of localised spatial weighting factors in the computations was found to improve its performance when registering DCE data [25]. In the current study, although the improvement of SEMI compared to Slicer that was noted in the clinical evaluation did not reach significance, the improvement in its CNR performance was significant. However, its improved ability to deal with motion may prove to be the most significant feature of this algorithm when implementing deformable registration into a routine clinical environment, where a reproducible and robust performance of such an image processing step is essential. This is particularly relevant for patients with compromised blood flow in the legs who often experience difficulty in keeping their legs steady for any length of time, and for whom strapping the legs together to prevent motion can be extremely uncomfortable and even painful. In this context, a deformable registration algorithm which can deal with large-scale motions is a highly desirable feature. 
Despite the fact that 3D deformable registration is a computationally intensive process, the Slicer implementation carried out this processing step in an average time of 54 seconds using a standard desktop computer. The longer time taken by the SEMI algorithm is a consequence of the additional computation steps required by this algorithm due to its incorporation of the spatial information into the registration process, designed to make it more accurate and robust against the intensity nonuniformities found in contrast-enhanced MR images. The additional time required by the SEMI algorithm stems from (i) the computation of the cost function and the derivatives, and (ii) the convergence speed of the optimisation. On-going work involves the implementation of the SEMI algorithm using parallel computing via a 1024-core graphical processing unit (GPU) system, which is expected to reduce the computation time to less than one minute. Indeed, the rapid pace of development of GPU systems, driven in recent years by the rapacious demand for graphics handling capabilities from the gaming industry, are beginning to be exploited for medical image processing applications [33] and further improvements in performance are to be expected.

There is growing interest in the development of MRA techniques which do not involve the use of contrast agents, for example phase-contrast angiography using bipolar flow-encoding gradients. However, these techniques have not gained widespread clinical use due to their long acquisition times and reduced contrast performance. As such, contrast-enhanced MRA studies involving the subtraction of pre- from post-contrast images remain ubiquitous in the clinical arena. Most such examinations focus on localised body areas where the symptom presents, for example only imaging the legs, and hence the time difference between the acquisition of the pre- and post-contrast image datasets, and hence the potential for movement artefacts, are reduced compared to that described in the current whole body study. Nevertheless, given the asymptomatic nature of atherosclerotic disease, scanning the whole body is preferable, and since MR technology is beginning to allow for the acquisition of high quality images in sufficiently fast times to follow the first pass of the contrast agent bolus through the vasculature, measures to deal with the inevitable mis-registration artefacts in the subtracted datasets need to be developed and critically assessed.

\section{Conclusions}

Image registration is a powerful post-processing tool that is used routinely in many areas of medical image analysis, although its use in CE-MRA is extremely limited due primarily to the inability of conventional registration algorithms to deal with the large signal intensity changes between the pre- 
and post-contrast enhanced images. In the current study, significant qualitative and quantitative improvements in the diagnostic image quality were measured for two recently-developed registration algorithms based on the normalised mutual information metric. The SEMI algorithm was also found to correct for large patient movements, upwards of $25 \mathrm{~mm}$, which is particularly relevant for the implementation of a registration processing step into a routine clinical protocol. These features demonstrate the potential for detecting subtle stenoses in arteries, which is particularly important in whole body studies aimed at detecting the extent of atherosclerotic disease. A further significant finding in this work was the serious potential for mis-diagnoses arising from an inappropriate choice of registration algorithm. In this case, an algorithm used in a previous CE-MRA study was shown to cause a dramatic degradation of the integrity of the vessels in the registered image datasets in all patients examined. Registration algorithms could be easily incorporated into the routine image acquisition and processing pipeline in MR scanners using the powerful computing hardware present therein.

\section{Acknowledgement}

This study was funded through an institutional grant to the CAMI centre from the Health Research Board (HRB), Ireland, who also funded a studentship for one of the authors (DC).

\section{References}

[1] Belch JJ, Topol EJ, Agnelli G, Bertrand M, Califf RM, Clement DL, et al. Critical issues in peripheral arterial disease detection and management: a call to action. Archives of internal medicine. 2003;163:884-92.

[2] Criqui MH, Langer RD, Fronek A, Feigelson HS, Klauber MR, McCann TJ, et al. Mortality over a period of 10 years in patients with peripheral arterial disease. The New England journal of medicine. 1992;326:381-6.

[3] Zheng ZJ, Sharrett AR, Chambless LE, Rosamond WD, Nieto FJ, Sheps DS, et al. Associations of ankle-brachial index with clinical coronary heart disease, stroke and preclinical carotid and popliteal atherosclerosis: the Atherosclerosis Risk in Communities (ARIC) Study. Atherosclerosis. 1997;131:115-25.

[4] Sen S, Lynch DR, Jr., Kaltsas E, Simmons J, Tan WA, Kim J, et al. Association of asymptomatic peripheral arterial disease with vascular events in patients with stroke or transient ischemic attack. Stroke; a journal of cerebral circulation. 2009;40:3472-7.

[5] Wang JY, Abdi N, Bakhadirov K, Diaz-Arrastia R, Devous MD. A comprehensive reliability assessment of quantitative diffusion tensor tractography. Neurolmage. 2012;60:1127-38.

[6] Jonsson $T$, Wennerberg $A B$, Forssberg $H$, Glover GH, Li TQ. An image registration strategy for multi-echo fMRI. Journal of magnetic resonance imaging : JMRI. 1999;10:154-8. 
[7] Hajnal JV, Saeed N, Oatridge A, Williams EJ, Young IR, Bydder GM. Detection of subtle brain changes using subvoxel registration and subtraction of serial MR-images. Journal of computer assisted tomography. 1995;19:677-91.

[8] Llado X, Ganiler O, Oliver A, Marti R, Freixenet J, Valls L, et al. Automated detection of multiple sclerosis lesions in serial brain MRI. Neuroradiology. 2012;54:787-807.

[9] Klein A, Andersson J, Ardekani BA, Ashburner J, Avants B, Chiang MC, et al. Evaluation of 14 nonlinear deformation algorithms applied to human brain MRI registration. Neurolmage. 2009;46:786-802.

[10] Shan ZY, Mateja SJ, Reddick WE, Glass JO, Shulkin BL. Retrospective Evaluation of PET-MRI Registration Algorithms. Journal of Digital Imaging. 2011;24:485-93.

[11] Slomka PJ, Baum RP. Multimodality image registration with software: state-of-the-art. European Journal of Nuclear Medicine and Molecular Imaging. 2009;36:44-55.

[12] Woods RP, Cherry SR, Mazziotta JC. Rapid automated algorithm for aligning and reslicing PET images. Journal of computer assisted tomography. 1992;16:620-33.

[13] Allen JS, Bruss J, Mehta S, Grabowski T, Brown CK, Damasio H. Effects of spatial transformation on regional brain volume estimates. Neurolmage. 2008;42:535-47.

[14] Menke J. Contrast-enhanced magnetic resonance angiography in peripheral arterial disease: improving image quality by automated image registration. Magnetic resonance in medicine : official journal of the Society of Magnetic Resonance in Medicine / Society of Magnetic Resonance in Medicine. 2008;60:224-9.

[15] Bergamino M, Bonzano L, Levrero F, Mancardi GL, Roccatagliata L. A review of technical aspects of T1-weighted dynamic contrast-enhanced magnetic resonance imaging (DCE-MRI) in human brain tumors. Physica Medica. 2014;30:635-43.

[16] Rueckert D, Sonoda LI, Hayes C, Hill DLG, Leach MO, Hawkes DJ. Nonrigid registration using freeform deformations: Application to breast MR images. IEEE transactions on medical imaging. 1999;18:712-21.

[17] West J, Fitzpatrick JM, Wang MY, Dawant BM, Maurer CR, Kessler RM, et al. Comparison and evaluation of retrospective intermodality brain image registration techniques. Journal of computer assisted tomography. 1997;21:554-66.

[18] Denton ER, Sonoda LI, Rueckert D, Rankin SC, Hayes C, Leach MO, et al. Comparison and evaluation of rigid, affine, and nonrigid registration of breast MR images. Journal of computer assisted tomography. 1999;23:800-5.

[19] Rohlfing T, Maurer CR, Bluemke DA, Jacobs MA. Volume-preserving nonrigid registration of MR breast images using free-form deformation with an incompressibility constraint. IEEE transactions on medical imaging. 2003;22:730-41.

[20] Li X, Dawant BM, Welch EB, Chakravarthy AB, Freehardt D, Mayer I, et al. A nonrigid registration algorithm for longitudinal breast $\mathrm{MR}$ images and the analysis of breast tumor response. Magnetic Resonance Imaging. 2009;27:1258-70.

[21] Tokuda J, Mamata H, Gill RR, Hata N, Kikinis R, Padera RF, et al. Impact of Nonrigid Motion Correction Technique on Pixel-Wise Pharmacokinetic Analysis of Free-Breathing Pulmonary Dynamic Contrast-Enhanced MR Imaging. Journal of Magnetic Resonance Imaging. 2011;33:968-73.

[22] Oguro S, Tokuda J, Elhawary H, Haker S, Kikinis R, Temparly CMC, et al. MRI Signal Intensity Based B-Spline Nonrigid Registration for Pre- and Intraoperative Imaging During Prostate Brachytherapy. Journal of Magnetic Resonance Imaging. 2009;30:1052-8.

[23] Mainardi LT, Passera KM, Lucesoli A, Potepan P, Setti E, Musumeci R. A method for dynamic subtraction MR imaging of the liver. BMC medical imaging. 2006;6:5.

[24] Viswanath S, Bloch BN, Genega E, Rofsky N, Lenkinski R, Chappelow J, et al. A comprehensive segmentation, registration, and cancer detection scheme on 3 Tesla in vivo prostate DCE-MRI. Medical image computing and computer-assisted intervention : MICCAI International Conference on Medical Image Computing and Computer-Assisted Intervention. 2008;11:662-9. 
[25] Zhuang X, Arridge S, Hawkes DJ, Ourselin S. A nonrigid registration framework using spatially encoded mutual information and free-form deformations. IEEE transactions on medical imaging. 2011;30:1819-28.

[26] Tanner C, Schnabel JA, Chung D, Clarkson MJ, Rueckert D, Hill DLG, et al. Volume and Shape Preservation of Enhancing Lesions when Applying Non-rigid Registration to a Time Series of Contrast Enhancing MR Breast Images. Proceedings of the Third International Conference on Medical Image Computing and Computer-Assisted Intervention: Springer-Verlag; 2000. p. 327-37.

[27] Rajaraman S, Rodriguez JJ, Graff C, Altbach MI, Dragovich T, Sirlin CB, et al. Automated registration of sequential breath-hold dynamic contrast-enhanced MR images: a comparison of three techniques. Magnetic Resonance Imaging. 2011;29:668-82.

[28] Hipwell JH, Penney GP, McLaughlin RA, Rhode K, Summers P, Cox TC, et al. Intensity-based 2-D3-D registration of cerebral angiograms. IEEE transactions on medical imaging. 2003;22:1417-26.

[29] Menke J. Improving the image quality of contrast-enhanced MR angiography by automated image registration: a prospective study in peripheral arterial disease of the lower extremities. European journal of radiology. 2010;75:e1-8.

[30] Willinek WA, Gieseke J, Conrad R, Strunk H, Hoogeveen R, von Falkenhausen M, et al. Randomly segmented central k-space ordering in high-spatial-resolution contrast-enhanced MR angiography of the supraaortic arteries: initial experience. Radiology. 2002;225:583-8.

[31] Herborn CU, Ajaj W, Goyen M, Massing S, Ruehm SG, Debatin JF. Peripheral Vasculature: WholeBody MR Angiography with Midfemoral Venous Compression-Initial Experience. Radiology. 2004;230:872-8.

[32] Yushkevich PA, Piven J, Hazlett HC, Smith RG, Ho S, Gee JC, et al. User-guided 3D active contour segmentation of anatomical structures: Significantly improved efficiency and reliability. Neurolmage. 2006;31:1116-28.

[33] Modat M, Ridgway GR, Taylor ZA, Lehmann M, Barnes J, Hawkes DJ, et al. Fast free-form deformation using graphics processing units. Computer methods and programs in biomedicine. 2010;98:278-84. 


\section{Figure Legends}

Figure 1: Typical positioning of the vessel (red) and background tissue (yellow) regions of interest (ROI) used for the contrast-to-noise ratio measurements. In four patients, the field of view necessitated drawing the ROI lower along the anterior tibial artery.

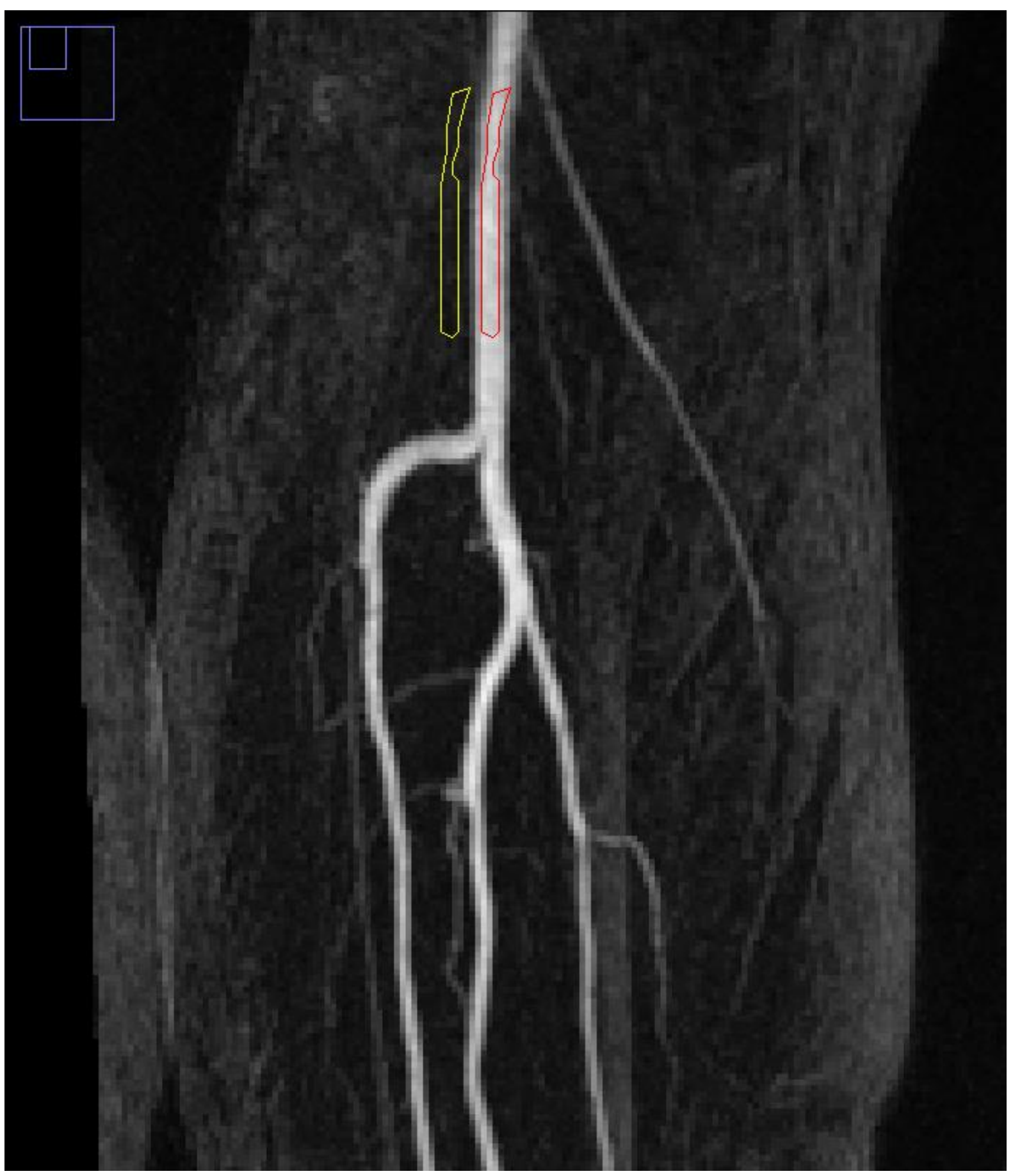


Figure 2: Example of the edge artefact introduced by all registration algorithms. Top row: manifestation of the artefact in the first slice of the subtracted image stack, together with the corresponding uncorrected (middle row) and corrected (bottom row) MIPs. The columns (left to right) show the images created using AIR, Slicer and SEMI.

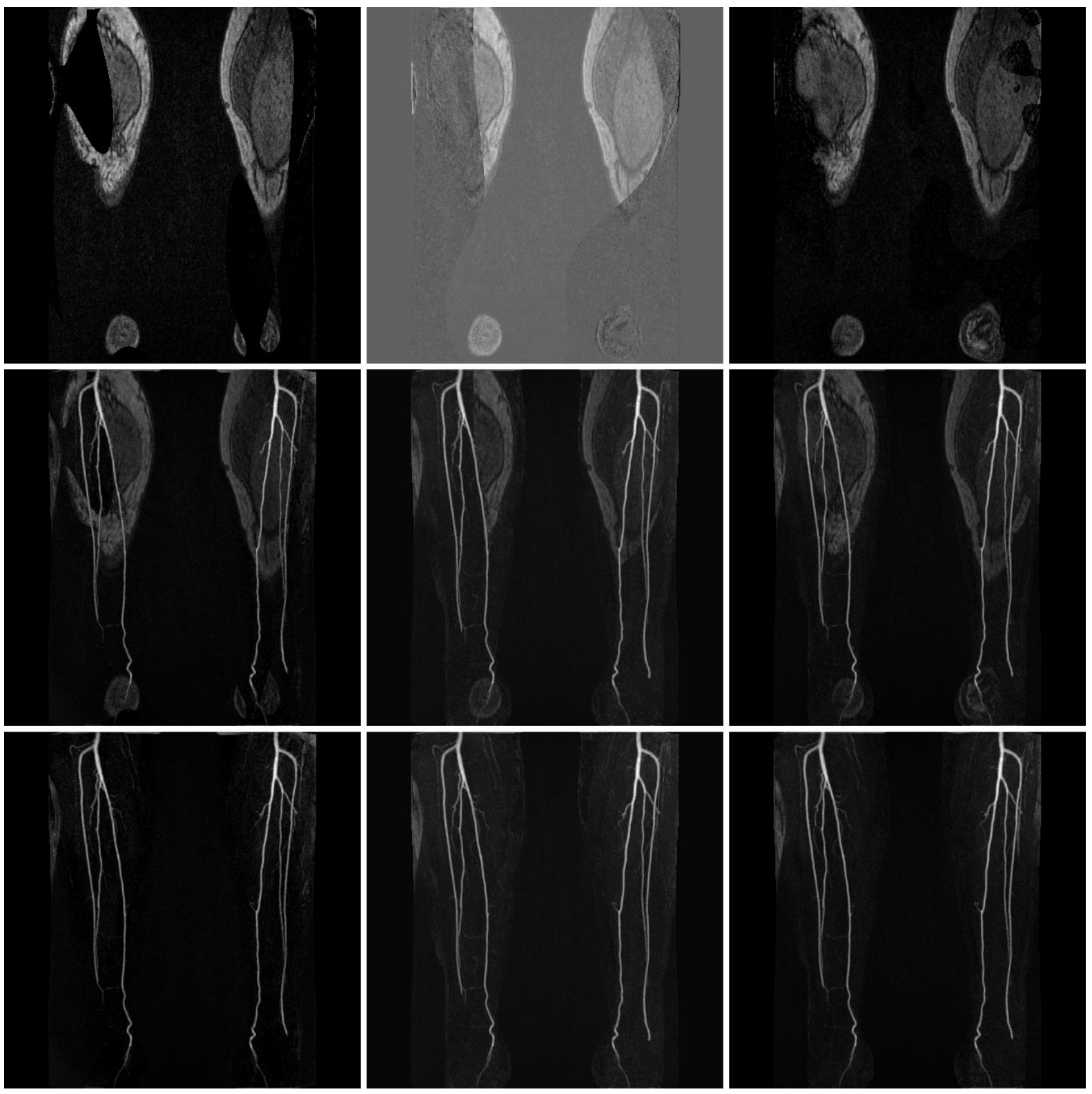


Figure 3: The diagnostic image quality was found by the four expert observers to be significantly improved following application of all three registration algorithms. The images were presented to the observers in a randomised, blinded manner; in this example, these MIP images represent (a) no registration, and registered with (b) AIR, (c) Slicer and (d) SEMI.
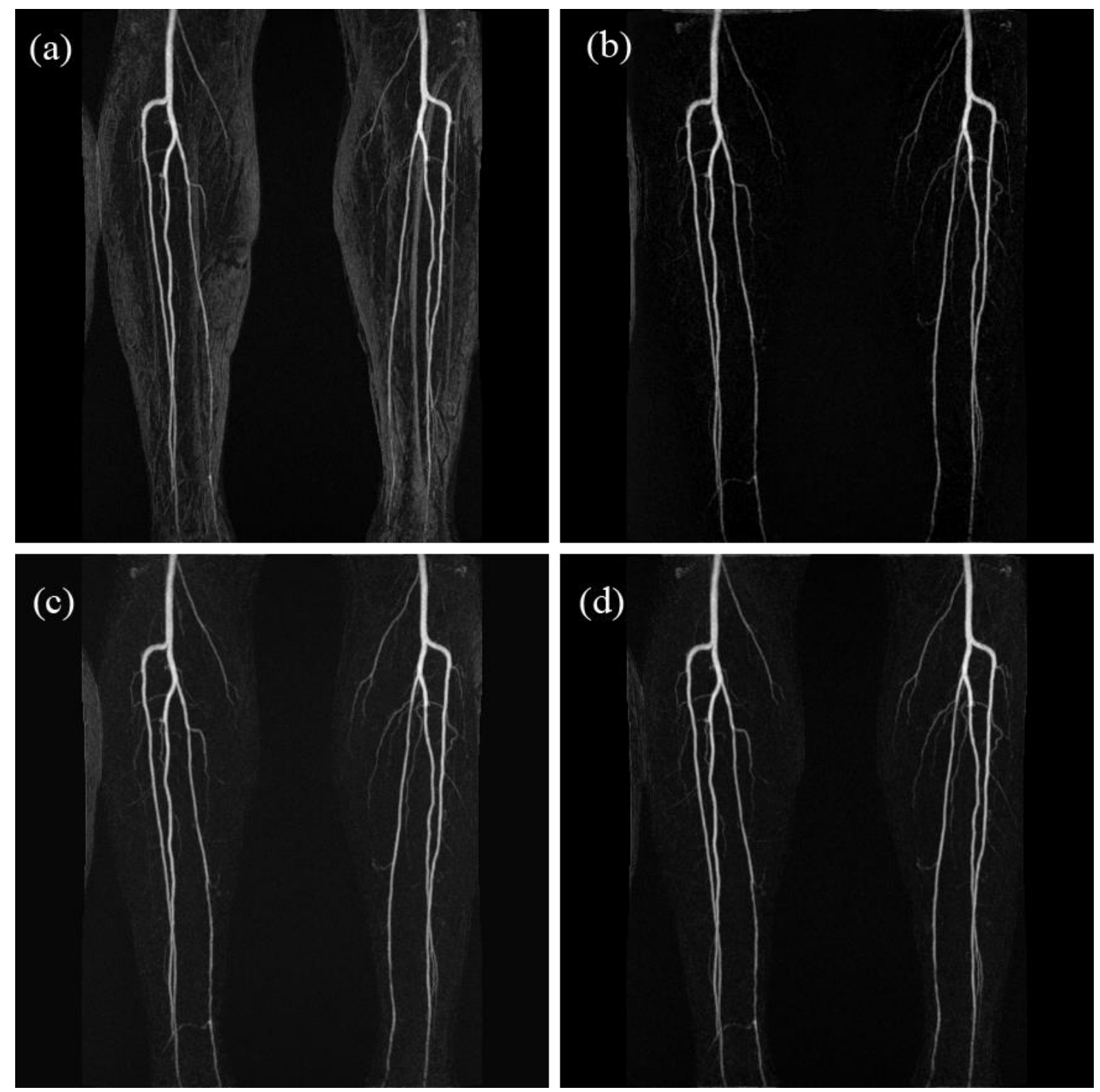
Figure 4: Comparison of the MIP images for the left leg for one patient with (a) no registration, and registered using (b) AIR, (c) Slicer, and (d) SEMI. The improved background tissue suppression achieved by the AIR algorithm is evident.

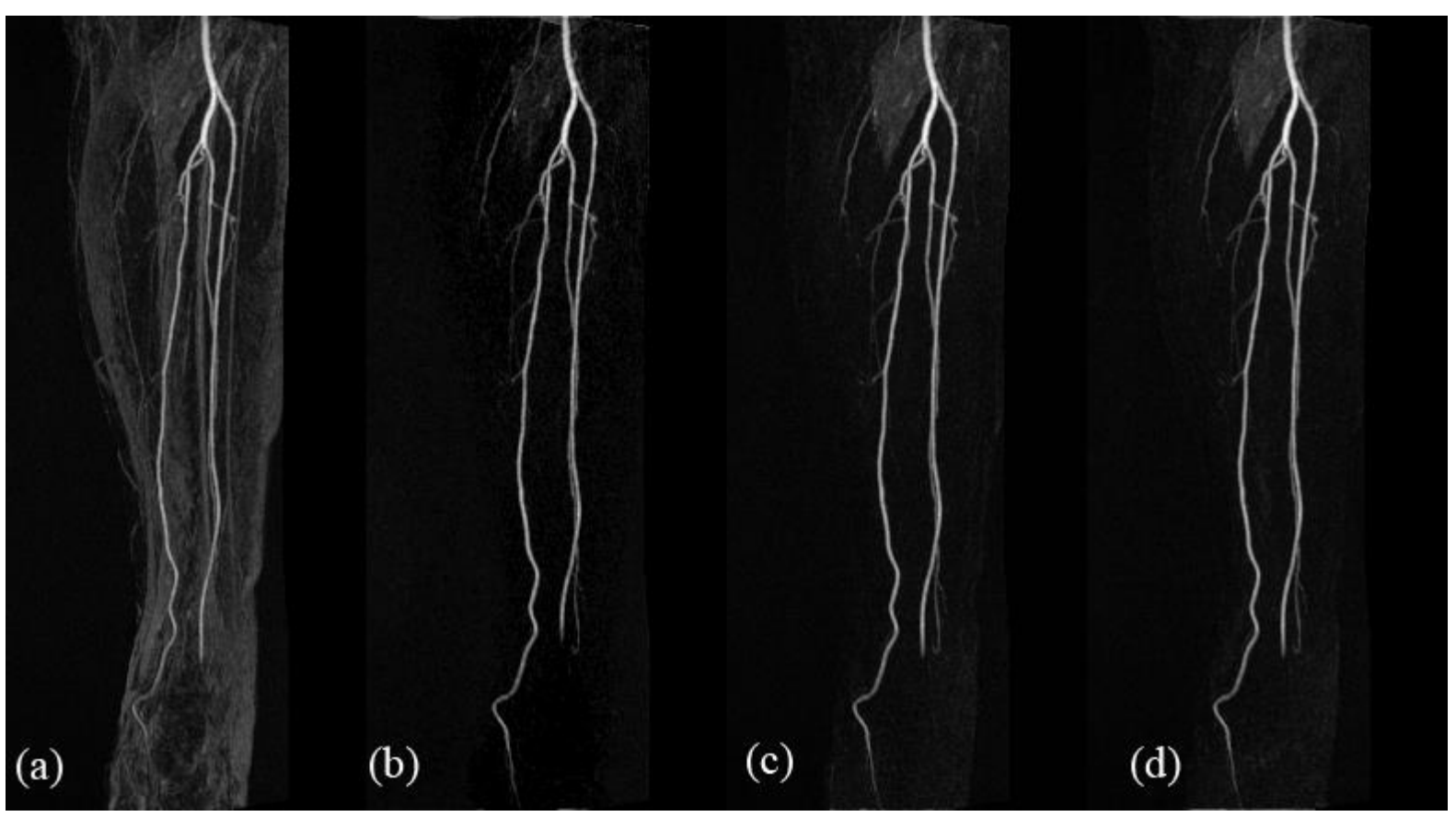


Figure 5: Images from one patient demonstrating the introduction of artefacts into the blood vessels including the loss of vessel integrity introduced by the AIR algorithm, manifest by the numerous signal drop-outs along the vessels (examples indicated by the arrows) which could be mis-diagnosed as stenoses: (a) no registration, and registered using (b) AIR, (c) Slicer, and (d) SEMI.

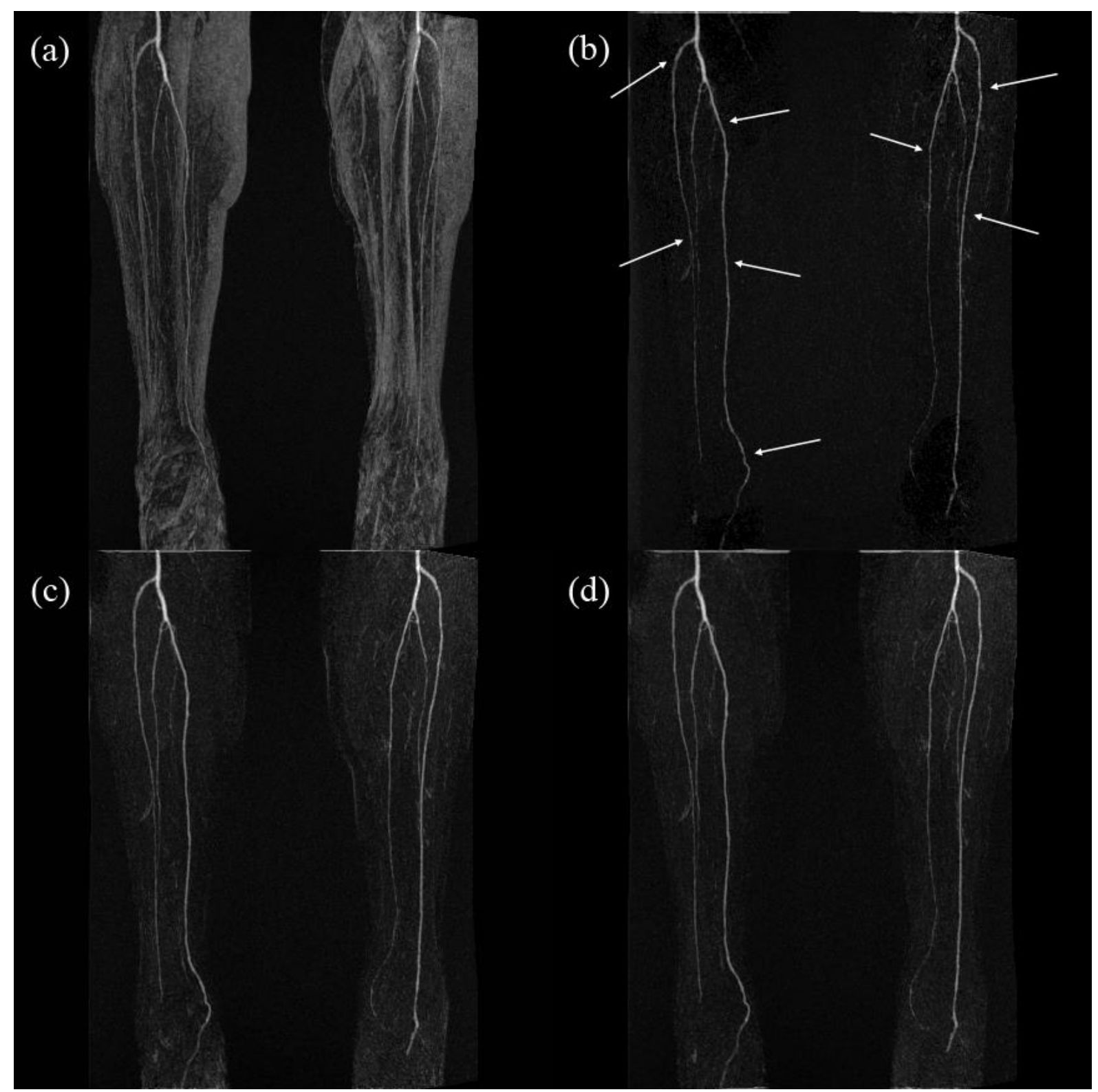


Figure 6: Surface-rendered view of a segmented vessel section at the location of the branching of the popliteal and anterior tibial arteries for the case of (a) no registration, and with registration using (b) AIR, (c) Slicer and (d) SEMI. The arrow indicates an artefactual narrowing of the vessel in the AIRgenerated image, which could give rise to a misdiagnosis in this patient.

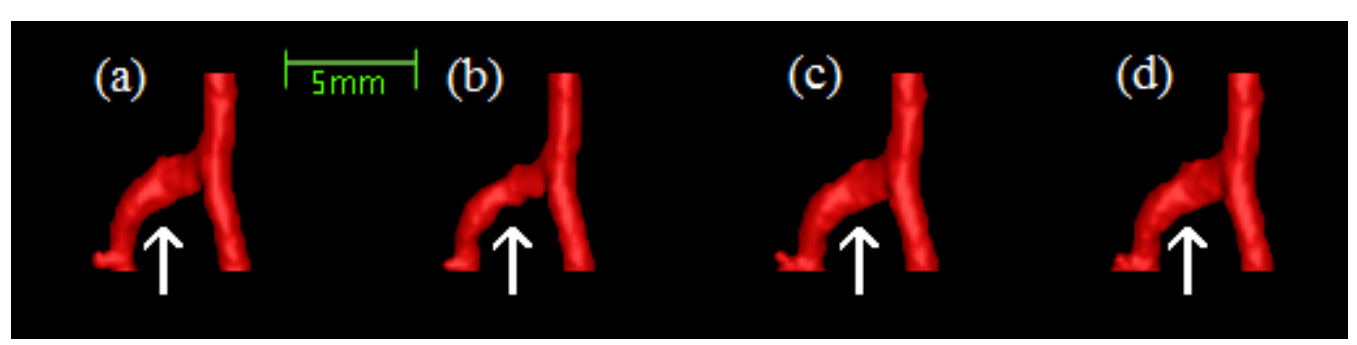


Figure 7: MIP images of the phantom subtraction images after registration by: (a) AIR following a lateral displacement (from left to right in this image) of $1 \mathrm{~mm}$, (b) Slicer following a lateral displacement of $15 \mathrm{~mm}$, and (c) SEMI a lateral displacement of $25 \mathrm{~mm}$. The solid white arrows show the position of the $1.6 \mathrm{~mm}$ diameter vessel which was removed by the AIR algorithm in (a) but not by the others. The spotting artefacts introduced by Slicer and SEMI are evident in both (b) and (c), while examples of the mirror vessel artefacts introduced by Slicer are illustrated by the arrow heads in (b). Note also the reduced overall background tissue suppression in (b), particularly towards the centre of the image.

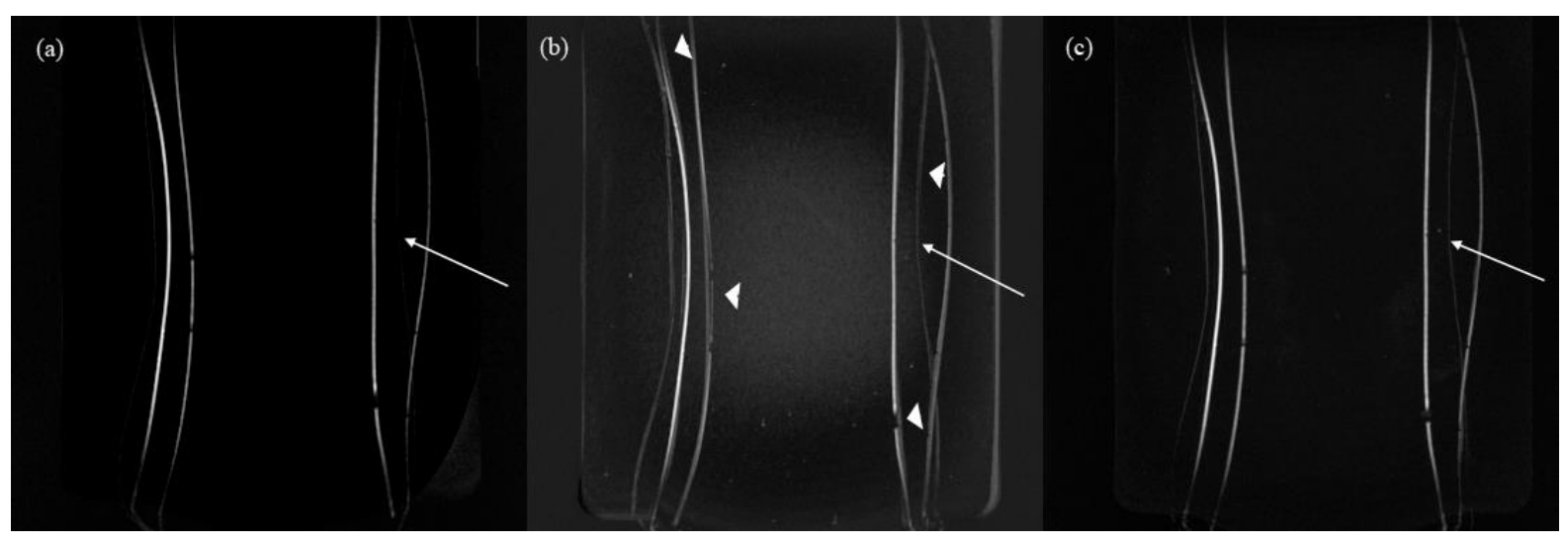

